\title{
BOTDA Sensing Employing a Modified Brillouin Fiber Laser Probe Source
}

\author{
Diego Marini $^{(\mathbb{1})}$, Marco Iuliano, Filippo Bastianini, and Gabriele Bolognini ${ }^{(0)}$, Member, IEEE
}

\begin{abstract}
A theoretical and experimental study has been carried out on a tunable dual pump-probe optical source for distributed Brillouin optical time-domain analysis (BOTDA). The developed source exploits a modified Brillouin ring laser technology and is capable of a tuning range of $\sim 200 \mathrm{MHz}$ without using phaselocked loop or optical sideband generation techniques, and exhibits a linewidth smaller than $2.5 \mathrm{MHz}$ and $\sim 0.5 \mathrm{~mW}$ power. In BOTDA experiments, the proposed source has demonstrated to be an efficient solution enabling distributed sensing over $10 \mathrm{~km}$ single mode fiber with a spatial resolution of $\sim 4 \mathrm{~m}$, and a strain and temperature resolutions of $\sim 10 \mu \varepsilon$ and $\sim 0.5^{\circ} \mathrm{C}$ respectively.
\end{abstract}

Index Terms-Brillouin scattering, fibers, nonlinear optics, optical fiber sensors.

\section{INTRODUCTION}

D ISTRIBUTED optical fiber sensors (DOFS) offer the possibility of monitoring several physical quantities along the optical length of the fiber in a distributed way through the analysis of the environment-dependent scattering suffered by the radiation travelling into the waveguide [1]. DOFS based on Brillouin technology enable distributed measurements of both strain and temperature, and constitute the most studied and used DOFS systems in civil engineering and geological applications [2].

Brillouin Optical Time Domain Analysis (BOTDA), as well as Brillouin Optical Frequency-Domain Analysis (BOFDA) schemes, exploit the temperature- and strain- dependence of the Brillouin frequency shift (BFS) of the back scattered radiation to reconstruct the spatial variation of the physical quantities under analysis over sensing length exceeding tens of kilometers [3]. In particular, the phonon population of a portion of material is strongly affected by the local temperature and is altered by the presence of mechanical deformations in accordance with the characteristic of material, silica for fibers. Such spatial dependence of the phonons leads to a variation in the spectrum, and

Manuscript received July 31, 2017; revised October 4, 2017; accepted November 2, 2017. Date of publication November 12, 2017; date of current version February 24, 2018. This work was supported by the European Union through Horizon 2020, the Framework Programme for Research and Innovation, under Project PULSe, Grant 737801. (Corresponding author: Gabriele Bolognini.)

D. Marini and G. Bolognini are with the Consiglio Nazionale delle Ricerche, IMM Institute, Bologna 40129, Italy (e-mail: marini@bo.imm.cnr.it; bolognini@bo.imm.cnr.it).

M. Iuliano is with the Consiglio Nazionale delle Ricerche, IMM Institute, Bologna 40129, Italy, and also with the University of Bologna, Bologna 40126, Italy (e-mail: iuliano@bo.imm.cnr.it).

F. Bastianini is with Sestosensor s.r.l., Bologna 40069, Italy (e-mail: fbi@sestosensor.it).

Color versions of one or more of the figures in this paper are available online at http://ieeexplore.ieee.org.

Digital Object Identifier 10.1109/JLT.2017.2772326 in particular in the central frequency, of the Brillouin scattered radiation which can be used to reconstruct the temperature and strain distribution [4]. In BOTDA schemes, the spatial variation of the BFS parameter is reconstructed by analyzing the temporal intensity change of a continuous-wave $(\mathrm{CW})$ radiation, the probe signal, counter-propagating with respect to a pulsed radiation, the pump signal [5]. If the probe frequency lies within the Brillouin gain spectrum of the pump, some power is transferred from the pump to the probe.

The efficiency of such transfer is given by the frequency difference between the probe signal and the Brillouin frequency shift which is dependent on the portion of fiber where the scattering takes place. The wavelength of probe signal is tuned so that the pump-probe frequency shift spans the whole Brillouin gain spectrum (BGS) of the pump. The BGS can be therefore reconstructed analyzing the temporal variation of the probe signal intensity in correspondence of each of the selected frequencies [6].

The maximum of BGS occurs when the frequency offset between the two optical waves equals the peak acoustic-phonon frequency, i.e., the BFS, which depends linearly on strain and temperature [1], providing a simple and effective mechanism to perform distributed sensing of these physical quantities along an optical fiber. The BFS change $\left(\Delta \nu_{B}\right)$ along the fiber can be expressed as a linear combination of temperature variation $(\Delta T)$ and strain variation $(\Delta \varepsilon)$, as follows:

$$
\Delta \nu_{\mathrm{B}}=C_{\nu_{\mathrm{B}} \varepsilon} \cdot \Delta \varepsilon+C_{\nu_{\mathrm{B}} T} \cdot \Delta T
$$

where $C_{\nu B \varepsilon}=0.048 \mathrm{MHz} / \mu \varepsilon$ and $C_{\nu B T}=1.07 \mathrm{MHz} /{ }^{\circ} \mathrm{C}$ are the BFS strain and temperature coefficients in silica fibers [5].

In BOTDA schemes, the techniques which are commonly employed for tuning the probe signal frequency are based on Phase-Locked Loop (PLL) [7] or optical side-band (OSB) generation method [8]. In PLL technique, probe and pump signals are generated by different sources, slave and master laser, respectively. The slave laser is wavelength locked to the master slave through a feedback system which allows tuning the frequency of the probe signal. In OSB method, the probe signal is modulated through an electro-optical modulator (EOM) which is driven at a frequency which corresponds to the desired wavelength shift. Both techniques require the introduction of costly devices in addition to those employed in interrogator and sensor blocks. In particular, the PLL-based systems use narrow linewidth sources as slave lasers, photodiodes and RF generators in the frequency-locking feedback systems. On the other hand, OSB technique requires wide bandwidth EOM and up-to $10 \mathrm{GHz}$ - electrical signal generator. Furthermore, the 
complexity of the systems implementing the mentioned techniques leads to issues in terms of stability, accuracy in the wavelength shift and long term performance which constitute a major obstacle to a large scale development of BOTDA systems. Recently, a considerable effort has been devoted to the development of cost-effective alternatives to PLL-technique and OSBmethod for the generation of pump-probe signals. Examples of novel schemes addressing such issue include BOTDA sensors employing a single optical source driven by a pulsed RF signals and passive optical filtering [9], systems based on time-division pump-probe generation by direct modulation of a laser diode through a arbitrary waveform generator [10] and sources which use the Stokes wave generated inside a highly nonlinear fiber (HNF) to generate the probe wave [11].

In this work we have studied a strain and temperature BOTDA sensing system which employs the recently developed pumpprobe source ${ }^{1}$ based on Brillouin ring laser (BRL) technology [12], [13]. Such technique has been shown to provide good performance in terms of tunable capabilities despite the simplicity of the scheme, in particular if we compare it with the mentioned complex techniques. In BRL scheme, the probe signal is generated through stimulated Brillouin scattering (SBS) of the pump signal implemented through a recirculation loop employing a modified Brillouin fiber ring laser (BRL). A PZT actuator is used to impart a tensile strain on the fiber ring and shift the BFS so that the frequency of the generated probe signal is accurately tuned. The linewidth and power of the signal generated through BRL technique show that such radiation can be effectively employed in pump-probe schemes. As shown below, the generated probe power $(0.5 \mathrm{~mW})$, the bandwidth (FWHM) values $(2.5 \mathrm{MHz})$ and tuning range $(\sim 200 \mathrm{MHz})$ allowed for a successful use in practical BOTDA schemes, demonstrating strain/temperature sensing over $10 \mathrm{~km}$ single-mode fiber.

\section{BRILLOUIN FIBER RING LASER}

The tunable source employed in the BOTDA experiments is essentially a modified version of a Brillouin ring laser (BRL) incorporating an Erbium Doped Fiber Amplifier (EDFA) within the cavity for boosting the Stokes signal and lowering the lasing threshold power. This type of laser applies Stimulated Brillouin Scattering (SBS) effect to produce a highly coherent radiation exploiting the resonance mechanism of the loop-based layout. In particular, the BFL laser transfers a certain amount of power from the master slave source to the back circulating Stokes signal which is inherently generated through Brillouin scattering with a frequency shift which is ultimately related to the silica phonon population. BRL takes advantage of the narrow-linewidth effect induced by the ring cavity to generate radiation with bandwidth, power, wavelength and tunability features suitable for BOTDA sensing [6]. Sources using amplification of pump and/or Stokes signal have been proposed in order to increase the relative low signal power which can be extracted from the cavity. For example, in [14] a hybrid Brillouin/erbium fiber laser has been demonstrated that uses both Brillouin gain in single-mode

\footnotetext{
${ }^{1}$ Patent pending
}

optical fiber and gain in erbium-doped fiber. In the hybrid BRL source employed in the proposed sensor, the amplification occurs within a EDFA only and no recirculation of pump signal is allowed due to the optical-circulator based configuration, as detailed later in the text.

The core of a general BRL laser is a low loss optical cavity realized forming a short piece of a single mode optical fiber into a closed ring by means of a 4-port directional optical coupler [15]. Properly adjusting the coupling ratio coefficients of the coupler and the length of the ring, it is possible to select a discrete set of resonant frequencies which are trapped inside the ring and transmit the others.

Under resonance condition, constructive interference between coherent components occurs at one output port, while destructive interference drops power radiation at the other output port.

In particular, being $k$ the coupling coefficient (or coupling ratio) and $\beta(\omega)$ the propagation constant of an optical fiber of length $L$, the following conditions are met at resonance frequencies [15]:

$$
\begin{aligned}
\beta L & =2 \pi m-\pi / 2 \\
k & =\left(1-\gamma_{0}\right) \exp (-2 \alpha L)
\end{aligned}
$$

where $m$ is any integer, $\gamma_{0}$ is the fractional coupler intensity loss and $\alpha$ is the fiber's amplitude attenuation coefficient,

The fiber resonator hence essentially behaves like a narrowband Fabry-Perot filter with the finesse $F$ given by:

$$
F=\pi \sqrt{k /}(1-k)
$$

with $k$ the resonant coupling coefficient defined in (2).

In cavity-based structures as ring lasers, the trapping of the radiation at resonance frequency can be exploited to enhance or trigger intensity-dependent effects. In particular, in BRL the great enhancement of the light intensity is exploited to trigger stimulated Brillouin scattering (SBS) [16]. Indeed the power of the circulating radiation grows until the dissipation in the resonator loop equals the input power, lowering the threshold input pump power for the SBS to occur.

The threshold input power is defined as the power of the input radiation which generates a gain of the Stokes line equal to the round trip loss and is given, as a function of fiber length, by equation [15]:

$$
P_{\text {thresh }}=2 \pi^{2} \frac{A_{e f f} / g}{F \cdot L}
$$

Equation (4), where $g$ is the fiber Brillouin gain coefficient and $A_{e f f}$ is the effective core area, highlights the importance of the resonator filtering quality: for high finesse values the threshold power can be significantly lower than the one observed in the open loop case. Actually, in the open-loop case, for fiber lengths greater than $20 \mathrm{~km}, \mathrm{P}_{\text {thresh }}$ values of tens of $\mathrm{mW}$ are commonly observed, while laser pump thresholds in the $\mu \mathrm{W}$ range can be attained for properly designed fiber ring laser schemes with meter-scale fiber lengths. This is evident considering the graph shown in Fig. 1, reporting the calculated power threshold for SBS onset in ring schemes based on a directional coupler following the above-mentioned model [15], 


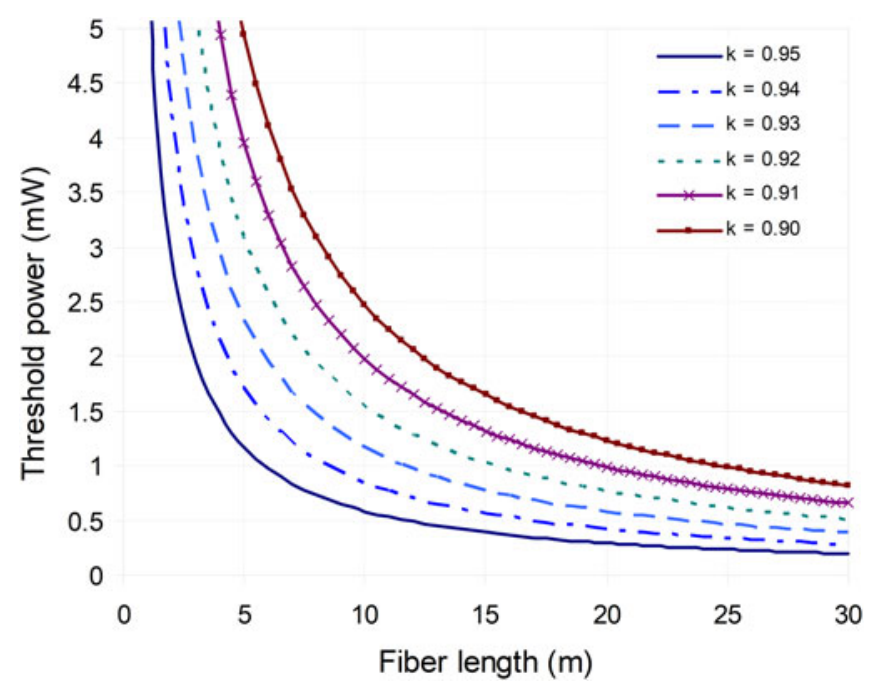

Fig. 1. Estimate of threshold power versus fiber length in a Brillouin fiber ring scheme for different values of directional coupling ratios $k$.

for different coupling ratios $k$ ranging from $90 \%$ to $95 \%$. In our model we used the Brillouin gain coefficient and effective area of the SMFs employed in our BOTDA experiments, i.e., $g=5.410^{-11} \mathrm{~m} / \mathrm{W}$ and $A_{\text {eff }}=6.010^{-12} \mu \mathrm{m}^{2}$. For increasing coupling ratios above $90 \%$ (and corresponding increasing resonator finesse values), the power threshold values drastically decrease, and can reach the sub-mW range even with fiber lengths of few tens of meters.

When the threshold power is reached, most of the pump power is transferred to the Stokes wave resulting in a lower gain experienced by the circulating radiation. In (4) the pump power depletion was neglected while for long length fiber resonator it should be taken into account since the power transfer from the seed and Stokes is relevant. Due to the length of the fiber coil $(>2 \mathrm{~km})$ under test in this work, a deviance from ideal behavior described by (4) is expected since the pump power should suffer a significant depletion at the end of a round trip [17].

In conventional BRL layout, as described above, a directional coupler is used to inject the seed radiation into the ring and to connect the termination of the loop with the output line. In the developed modified BRL laser, we have employed a 3 ports optical circulator where one port injects radiation into the ring whose terminations are connected through port 2 and 3. Such configuration allows the back-circulating Stokes signal, generated by Brillouin Scattering of the pump signal, to recirculate, resulting in an enhancement of its power. On the other hand the pump power, whose power has been depleted by the propagation loss and SBS, is tapped by the circulator. Therefore, the circulator based proposed solution provides a resonance structure for the Stokes signal only.

The scheme for the modified Brillouin fiber ring is depicted in Fig. 2. A distributed feedback laser (DFB) laser ( $\sim 1.25 \mathrm{MHz}$ linewidth) has been employed for generating the pump light. The Brillouin pump, centered around $\lambda=1.55 \mu \mathrm{m}$, is injected in single mode fiber (SMF) through port 1 on an optical circulator. A bi-directional Erbium Doped Fiber Amplifier (BEDFA) [18] is then placed in order to boost the seed power and

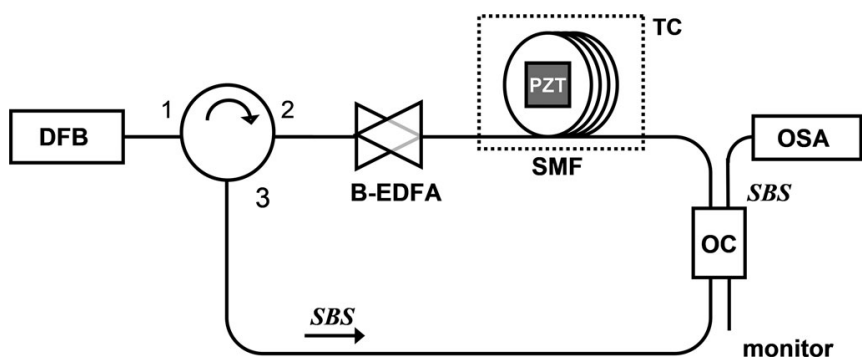

Fig. 2. Brillouin ring laser set-up. DFB: distributed feedback laser, B-EDFA: bi-directional Erbium-doped fiber amplifier, SMF: single-mode fiber, TC: temperature-controlled case, OC: optical coupler, OSA: optical spectrum analyzer.

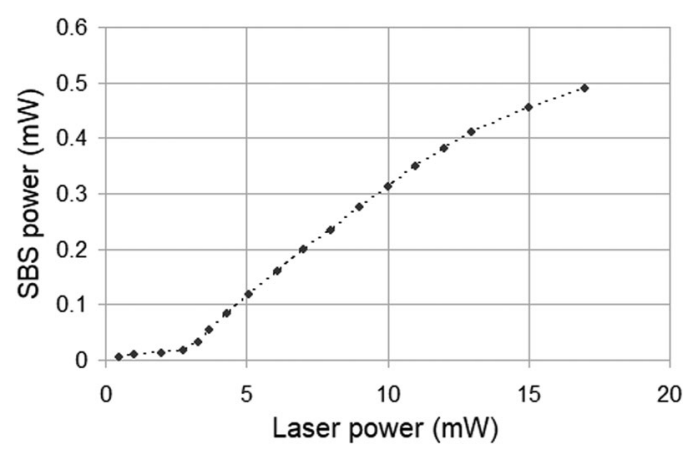

Fig. 3. Measured Brillouin probe power versus input pump seed power.

amplifying the generated SBS light, then effectively lowering the threshold level and enhancing the system efficiency. Note that no significant power oscillations were observed for gain values lower than $\sim 15 \mathrm{~dB}$. The Brillouin Scattering product Stokes line, together with the Rayleigh signal of the pump, propagating in the opposite direction of the seed, are reintroduced in the loop from port 2 through port 3. A portion of generated SBS backward-propagating radiation is coupled out from the ring cavity by using an optical coupler (OC, $95 / 5$ splitting ratio), and employed in BOTDA systems, or coupled into an optical spectrum analyzer (OSA) for source characterization. The BRL fiber has been placed in a temperature-controlled case (TC) for temperature stabilization avoiding unwanted frequency drifts. Initially we have evaluated the lasing efficiency of the novel BRL monitoring the power of the generated SSBS signal power. In particular, part of the circulating SBS signal has been extracted through a 95/5 directional coupler and the power of the radiation has been measured through a power meter.

Fig. 3 shows the extracted SBS power versus input pump power, indicating an threshold of $\sim 2 \mathrm{~mW}$ for onset of SBS probe, and a maximum output probe power of $\sim 0.5 \mathrm{~mW}$ with the available input pump power $(17 \mathrm{~mW})$, which is well suitable for implementing BOTDA [19].

Beside the power of the generated signal, its linewidth is another fundamental parameter to evaluate the possible use of the BRL signal in BOTDA schemes signal. Probe light linewidth should be narrow enough to allow for a correct reconstruction of BGS without affecting the sensor resolution (therefore it should be much narrower than BGS linewidth). In order to assess the validity of our proposed source, then it is important to reliably 


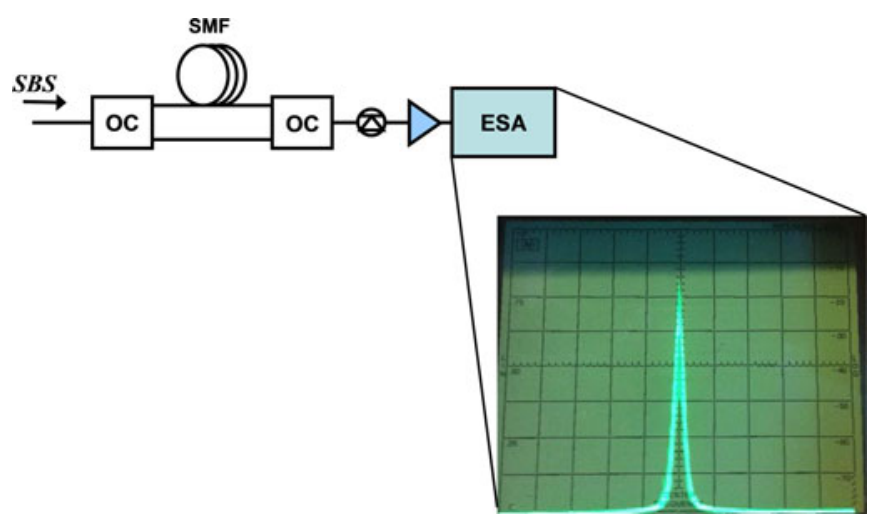

Fig. 4. Scheme of delayed self-heterodyne detection and electrical spectrum of extracted probe light after photo-detector and ESA.

characterize the probe source linewidth. We employed a self heterodyne technique [20], providing an accurate characterization for MHz-wide sources. In such a technique, represented in Fig. 4, the source light is split into two arms through an OC. A delay line in one OC arm (longer than expected source coherence length) ensures the two beams are uncorrelated at the second $\mathrm{OC}$, and output spectrum impinging on a photo-detector becomes essentially a self-convolution of laser spectrum providing a worst-case laser linewidth estimate. The spectrum distribution of the signal has been hence converted into an electric signal employing the delayed self-heterodyne method by a optical spectral linewidth test (OSLT) and observed through an high resolution $(<500 \mathrm{kHz})$ electrical spectrum analyzer (ESA) while the extracted power is measured by means of an optical power meter (OPM).

Fig. 4 also shows the measured linewidth estimation of the extracted SBS probe light from delayed self heterodyne technique into the ESA (10 MHz/freq. div), providing a worst-case estimate in terms of its convolution. Its full-width half maximum (FWHM) is measured to be $\sim 2.5 \mathrm{MHz}$, indicating a value which can be considered as about 0.1 the BGS linewidth, thus allowing for an accurate BGS reconstruction in BOTDA and strain/temperature sensing without affecting the measurand resolution.

It is worth noting that the attained linewidth value is twice the one of the seed radiation: this implies that the BRL laser generates a frequency-shifted signal with, approximately, the same FWHM order as the pump, despite the typical BGS spectral width is an order of magnitude wider (15-20 MHz). This effect can be related to the resonance mechanism of the ring cavity which acts as a narrow linewidth filter for the Stokes signal, leading to an effective BGS much narrower than the one observed in silica.

Finally, an assessment of intensity oscillations in the BRL source has been carried out. Actually, expected fluctuations in fiber lasers are typically higher than in integrated-photonics diode lasers essentially because of long fiber cavity lengths, resulting in oscillations and reduced stability due to onset of cavity mode hopping, pump-signal noise transfer and other effects [21]. In order to evaluate the power fluctuations of the BRL source we measured the relative intensity noise (RIN) value. The

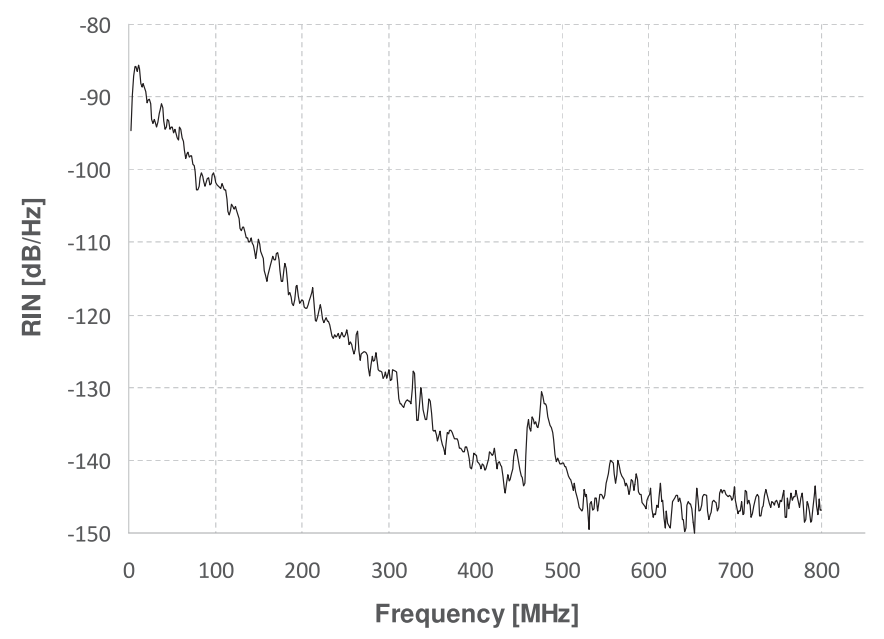

Fig. 5. Measured spectral RIN characteristics for the BRL source.

RIN is defined as the power spectral density of the time-varying signal power fluctuations $\delta \mathrm{P}(\mathrm{t})$ :

$$
R I N(\omega)=\frac{1}{\bar{P}^{2}} \int<\delta P(t) \delta P(t+\tau)>\exp (-i \omega \tau) d \tau
$$

where $\bar{P}$ is the average of the optical power and $<\cdot>$ denotes the average over a large number of measurements. We carried out RIN measurements on the BRL light using a fast PIN InGaAs photodetector (10 GHz bandwidth), acquiring the power spectrum $\sigma^{2}(\omega)$ through an electrical spectrum analyzer ESA (working up to $15 \mathrm{GHz}$ ) and subtracting thermal an shot noise terms [22].

The results of the RIN spectral measurements up to $800 \mathrm{MHz}$ are reported in Fig. 5. The graph in Fig. 5 shows that the RIN of the BRL is higher at lower frequencies. The low-frequency maximum values are observed at frequencies of $\sim 10-15 \mathrm{MHz}$ where RIN is about $-90 \mathrm{~dB} / \mathrm{Hz}$; the observed RIN decreases (approximately linearly) at higher frequencies until the 400$500 \mathrm{MHz}$ frequency range. Beyond $500 \mathrm{MHz}$ frequencies, RIN value is low and approximately constant with an average value of about $-145 \mathrm{~dB} / \mathrm{Hz}$. Such behavior is in accordance with theoretical and experimental results: fiber lasers such as those based on Brillouin and Raman effects, are known to exhibit higher RIN values with respect to diode laser pump seed, especially at lower frequencies [21].

\section{BOTDA SENSOR EXPERIMENT}

We then implemented a BOTDA sensor scheme with the optimized BRL tunable probe source. The experimental set-up used for the BOTDA sensor is reported in Fig. 6. One portion of power (approximately $30 \%)$ from a DFB laser $(1.55 \mu \mathrm{m}$ center wavelength, $\sim 1.25 \mathrm{MHz}$ linewidth), is used to generate the $\mathrm{CW}$ probe signal through the BRL source described in Section II (2.1 km fiber length, $3 \mathrm{dBm}$ input power). The remaining laser power is used as pump signal in the BOTDA scheme, after being amplified by an Erbium-doped fiber amplifier.

A Mach Zehnder intensity optical modulator module $(10 \mathrm{GHz}$ bandwidth, built-in polarization controller) driven by an RF 


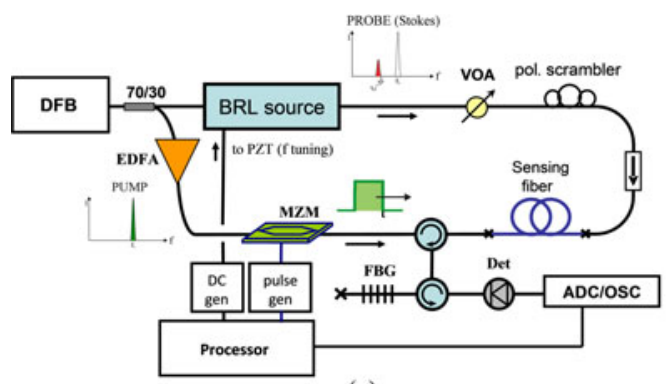

(a)

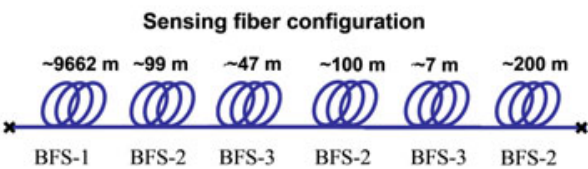

(b)

Fig. 6. (a) Set-up of the BOTDA sensor system employing the developed BRL light source. (b) Sensing fiber configuration detailing the different BFS characteristics (BFS-1 to BFS-3) of fiber spools.

pulse generator, modulates the intensity of the pump signal so that pulses of the order of nanoseconds are generated (40 ns pulse width, $\mathrm{RF}$ square waveform pulses, $7 \mathrm{kHz}$ repetition rate). Both the pulse generator and the DC-voltage generator used for the PZT actuators are computer-controlled. The $\mathrm{CW}$ probe signal and the pulsed pump signal are coupled with counterpropagating directions into the sensing fiber. The probe signal generated by the BRL source is coupled to a variable optical attenuator (VOA) in order to adjust the optical power ensuring optimal values (around $-2 \mathrm{dBm}$ ) avoiding nonlinear and pump depletion effects [23], and then passes through a polarization scrambler and optical isolation avoiding residual pump light leakage into the BRL module.

The probe signal after fiber propagation is collected at one end of the sensing fiber through an optical circulator and coupled into a $100 \mathrm{MHz}$-bandwidth photo-detector, after an optical filtering stage where the probe (Stokes) line is selected through reflection by a narrowband fiber Bragg grating $(\mathrm{FBG},<0.2 \mathrm{~nm}$ ) and a second optical circulator. Data acquisition is accomplished through a fast analog-to-digital converter (ADC) and subsequent data processing to reconstruct the BGS.

The frequency of the probe signal to reconstruct the fiber BGS is adjusted imparting an amount of tensile strain on the fiber ring coil, through the PZT actuators. Actually, as described in the previous Section, the shift between the pump seed and the BRL-generated probe inherently equals the BFS of the single mode fiber employed in the BRL cavity. Such signals can be therefore employed in BOTDA schemes requiring the presence, in the sensing element, of both the pump signal and a probe signal having a frequency within the Brillouin gain.

In order to probe all the Brillouin gain spectrum of the sensing fiber and reconstruct it as a function of the position, a large tuning range in probe frequency must be looked after, to measure BGS under different fiber strain and temperature conditions. In the proposed scheme the tuning is accomplished through the employment of piezo actuators (PZT) acting on the Brillouin ring fiber coil. The PZT actuators, driven by DC-voltage generator, impart tensile strain (up to $\sim 4 \mathrm{~m} \varepsilon$ ) and enable a large probe tuning range over $\sim 200 \mathrm{MHz}$, allowing one to monitor fiber temperature variations up to $150{ }^{\circ} \mathrm{C}$ and strain variations in the $m \varepsilon$ range.

As a piezo actuator, we employed a PZT fiber stretcher consisting of a high-voltage PZT ring where BRL fiber coil

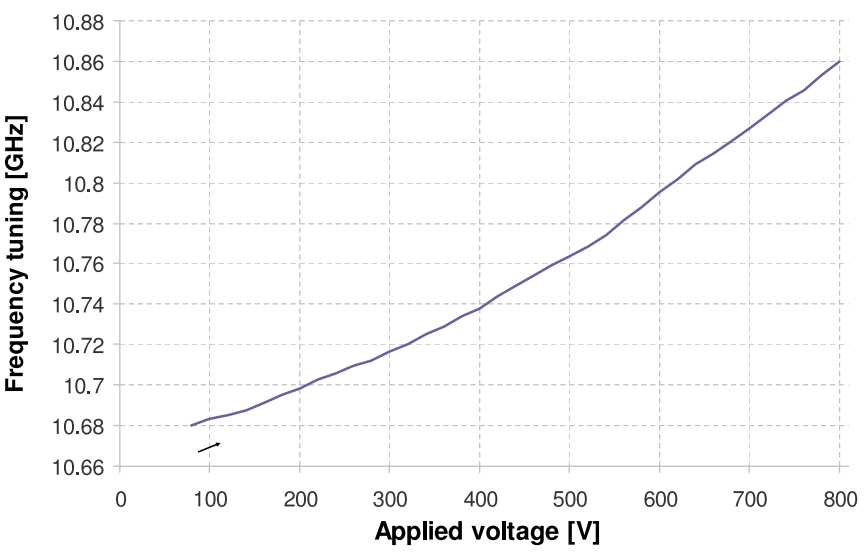

Fig. 7. BRL frequency tuning (with respect to pump wavelength) for increasing PZT voltage.

is placed. In wavelength tuning we acted uni-directionally by increasing PZT voltage ensuring $2 \mathrm{MHz}$ steps and avoiding possible hysteresis effects. The observed behaviour of PZT in our laboratory experiment was stable and repeatable. The output of BRL probe frequency vs applied voltage was employed to accurately estimate the BRL wavelength and is reported in Fig. 7, exhibiting a deviation from linear behaviour which is typical of PZT stretchers.

In order to emulate the presence of temperature gradients and/or mechanical strain, the employed sensing fiber is given by several spools of single mode fibers having different BFS values, for a total of $\sim 10 \mathrm{~km}$ SMF length.

The length of each fiber spool is shown in Fig. 6(b); in particular two very short spools of SMF (47 m and $7 \mathrm{~m}$ ) exhibit BFS values (i.e., BFS-3) which are down-shifted by about $30 \mathrm{MHz}$ from the initial long fiber spool BFS (BFS-1), and are interleaved with $\sim 100 \mathrm{~m}$ fiber spools whose BFS (BFS-2) is $\sim 20-\mathrm{MHz}$ upshifted with respect to initial fiber, providing a valid assessment for spatially-resolved sensing estimation in case of hot spots or short strained fiber sections. Merging all the acquired probe traces (time averaged, 1024 averages) in correspondence of different probe frequencies, and converting the timescale into the corresponding fiber position, we obtained the full BGS spectra for each fiber location.

In Fig. 8(a) we have reported the obtained 3-dimensional BGS spectrum for the last few kilometers of the sensing fiber, as a 


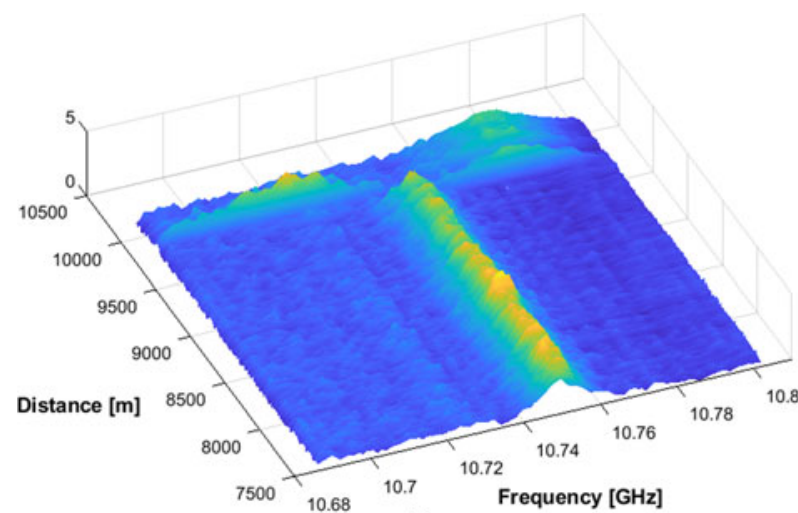

(a)

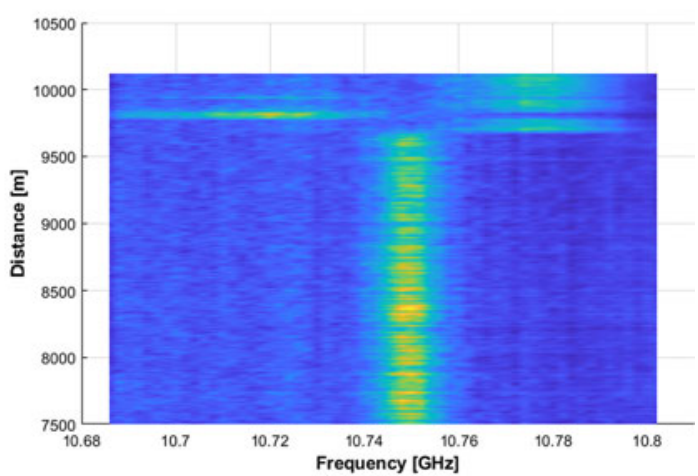

(b)

Fig. 8. (a) 3D and (b) top view (Contour plot) of the measured Brillouin gain spectrum (BGS) versus fiber distance for last sensing fiber kilometers of Fig. 6(b) spools configuration.

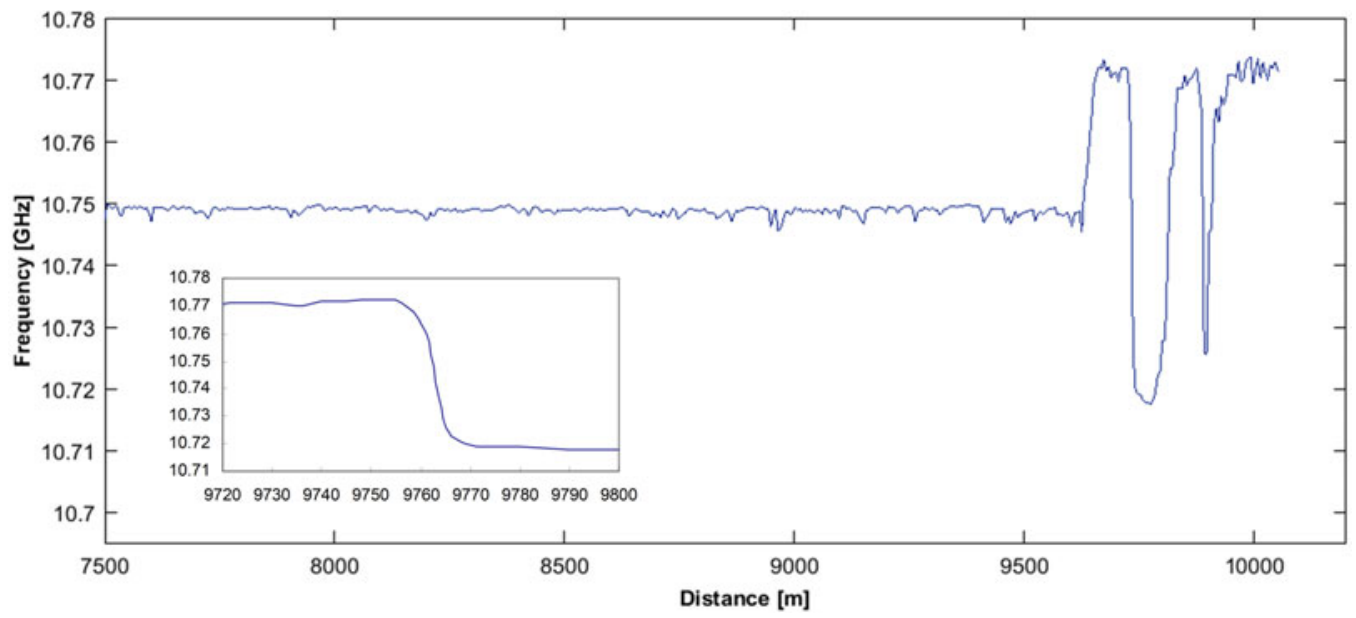

Fig. 9. Measured Brillouin frequency shift (BFS) versus distance for last fiber kilometers; Inset: zoom of frequency step between fiber spools BFS-2 and BFS-3 around a distance of $9760 \mathrm{~m}$.

function of the distance. The fluctuations appear to be mainly related to the intensity noise of the BRL probe light assessed at the end of Section II. The blue- and red-shift of the BGS can be neatly observed in the last meters in correspondence of the presence of different spools of sensing fibers, as also evident from Fig. 8(b) reporting, in a color plot, the BGS vs fiber length (top view of Fig. 8(a)). As it can be also observed, in addition to BGS values, also the BGS linewidths, lying in the $10-20 \mathrm{MHz}$ range, of different spools coming from different manufactures can differ significantly.

In the assessment of the use of proposed BRL for BOTDA it is also important to directly ensure that the generated probe features, namely power, frequency stability with respect to pump wavelength, tunability, and inherent linewidth, enable a correct discrimination of different BFS values (i.e., temperature-strain variations), and, in spite of higher low-frequency intensity noise values than diode lasers (as expected in fiber lasers), provide acceptable resolution levels.

We then calculated the BFS parameters through a Lorentzian fitting of the gain spectra for each spatial position. The results are shown in Fig. 9 where the obtained BFS values (in $\mathrm{MHz}$ scale) are reported as a function of the distance from the end fiber for the last few fiber kilometers.

The behavior of the BFS clearly matches the one expected from fiber spool configuration; in particular, in correspondence of the position of the fiber spool (BFS-2) whose BFS is $\sim 20-\mathrm{MHz}$ up-shifted with respect to initial fiber, an abrupt increase of measured value is clearly observed. The same happens for the BFS-3 fiber spools. Even the presence of the short $7 \mathrm{~m}$ fiber spool at the end of $10 \mathrm{~km}$ distance is revealed by the graph. The spatial resolution of the implemented BOTDA (estimated as $10 \%-90 \%$ step response in measured BFS traces) was equal to $\sim 4$ meters at the fiber end, as it can be seen from the zoom in Fig. 9 inset highlighting the measured frequency step between fiber spools BFS-2 and BFS-3 around a $9760 \mathrm{~m}$ distance.

We then calculated the root mean square $(\mathrm{rms})$ of BFS traces (as the one reported in Fig. 9) providing the frequency resolution attained by BOTDA sensing with our proposed BRL-based probe source. The obtained rms has been smaller than $\sim 0.5$ $\mathrm{MHz}$ throughout the fiber length, representing an attained resolution in terms of temperature and strain equivalent to $\sim 0.5^{\circ} \mathrm{C}$ and $\sim 10 \mu \varepsilon$, respectively. 
The developed source hence appears an efficient solution for generating a probe signal with inherent frequency locking to pump wavelength, possessing an adequate power, a low threshold behaviour, a narrow linewidth and tunability features which can be useful for distributed sensing.

\section{CONCLUSION}

In conclusion, we have successfully applied our proposed BRL-based optical source to distributed temperature and strain measurements in BOTDA-based sensing. The dual pump-probe optical source, based on a modified Brillouin fiber ring laser, can be a cost-effective solution to overcome the limitations affecting conventional BOTDA systems (given by expensive and complex components and techniques) by providing a tunable, pump-locked narrow-wavelength probe signal with adequate output power. Although characterized by moderated RIN values at low frequency, as typical for fiber lasers, the BRL source showed an adequate probe output power $(\sim 0.5 \mathrm{~mW})$, large tunability (up to $\sim 200 \mathrm{MHz}$ ), a narrow linewidth $(<2.5 \mathrm{MHz}$ ) and inherent locking to pump wavelength, in addition to a low threshold behaviour. This feature allowed us to successfully set-up a cost-effective BOTDA sensor scheme providing down to 4 meter spatial resolution over $\sim 10 \mathrm{~km}$ single mode optical fiber with strain and temperature resolutions of $\sim 10 \mu \varepsilon$ and $\sim 0.5^{\circ} \mathrm{C}$ respectively, which represents a validation of the possible use of the pump-probe signals generation technique as an efficient alternative to the commonly employed techniques based on phase-locked loop or optical side-band generation methods in BOTDA-based sensing.

\section{REFERENCES}

[1] A. Barrias, J. R. Casas, and S. Villalba, "A review of distributed optical fiber sensors for civil engineering applications," J. Sensors, vol. 16, no. 5, May 2016, Art. no. 748.

[2] T. Horiguchi, K. Shimizu, T. Kurashima, M. Tateda, and Y. Koyamada, "Development of a distributed sensing technique using Brillouin scattering," J. Lightw. Technol., vol. 13, no. 7, pp. 1296-1301, Jul. 1995.

[3] D. Garus, "Distributed sensing technique based on Brillouin optical-fiber frequency-domain analysis," Opt. Lett, vol. 21, no. 17, pp. 1402-1404, Sep.1996.

[4] X. Bao, J. Dhliwayo, N. Heron, D. J. Webb, and D. A. Jackson, "Experimental and theoretical studies on a distributed temperature sensor based on Brillouin scattering," J. Lightw. Technol., vol. 13, no. 7, pp. 1340-1348, Jul. 1995.

[5] C. A. Galindez-Jamioy and J. M. López-Higuera, "Brillouin distributed fiber sensors: An overview and applications," J. Sensors, vol. 2012, Aug. 2012, Art. no. 204121.
[6] M. A. Soto et al., "Simplex-coded BOTDA fiber sensor with $1 \mathrm{~m}$ spatial resolution over a $50 \mathrm{~km}$ range," Opt. Lett., vol. 35, no. 2, pp. 259-261, Jan. 2010.

[7] A. W. Brown et al., "Brillouin scattering based distributed sensors for structural applications," J. Intell. Mater. Syst. Struct., vol. 10, no. 4, pp. 340-349, Apr. 1999.

[8] M. Nikles, L. Thévenaz, and P. A. Robert, "Simple distributed fiber sensor based on Brillouin gain spectrum analysis," Opt. Lett., vol. 21, no. 10, pp. 758-760, May 1996.

[9] H. Iribas et al., "Cost-effective brillouin optical time-domain analysis sensor using a single optical source and passive optical filtering," J. Sensors, vol. 2016, 2016, Art. no. 8243269

[10] K. Yong Song and S. Yang, "Simplified Brillouin optical time-domain sensor based on direct modulation of a laser diode," Opt. Express, vol. 18 no. 33, pp. 24012-24018, Nov. 2010.

[11] M. Bravo et al., "Application of remote power-by-light switching in a simplified BOTDA sensor network," Sensors, vol. 13, no. 10, pp 17334 17344, Oct. 2013.

[12] F. Bastianini, D. Marini, and G. Bolognini, "Modified Brillouin ring laser technology for Brillouin-based sensing," in Opt. Proc. SPIE 9634, 24th Int. Conf. Opt. Fiber Sensors, Curitiba, Brazil, 2015, Paper 96345E.

[13] M. Iuliano et al., "BOTDA sensing system employing a tunable low-cost Brillouin fiber ring laser pump-probe source," in Proc. SPIE 10323, 25th Int. Conf. Opt. Fiber Sensors, Jeju, South Korea, 2017, Paper 1032395.

[14] G. J. Cowle and D. Y. Stepanov, "Hybrid Brillouin/erbium fiber laser," Opt. Lett, vol. 21, no. 16, pp. 1250-1252, Aug. 1996.

[15] L. F. Stokes, M. Chodorow, and H. J. Shaw, "All-single-mode fiber resonator," Opt. Lett, vol. 7, no. 6, pp. 288-290, Jun. 1982.

[16] M. A. Hambali, "Reduction of brillouin threshold by circulating spontaneous brillouin stokes in ring cavity," in Proc. Topical Meeting Lasers Optoelectron., 2009, pp. 754-755.

[17] P. Bayvel and I. P. Giles, "Evaluation of performance parameters of singlemode all-fiber Brillouin ring lasers," Opt. Lett., vol. 14, no. 11, pp. 581583, Jun 1989.

[18] E. Desurvire, Erbium-Doped Fiber Amplifiers: Principles and Applications. New York, NY, USA: Wiley, 1994.

[19] D. Yongkang, L. Chen, and X. Bao, "Time-division multiplexing-based BOTDA over 100km sensing length," Opt. Lett, vol. 36, no. 2, pp. 277279, Jan. 2011.

[20] P. Horak and W. H. Loh, "On the delayed self-heterodyne interferometric technique for determining the linewidth of fiber lasers," Opt. Express, vol. 14, no. 9, pp. 3923-3928, May 2006.

[21] J. Geng and J. Shibin Jiang, "Pump-to-Stokes transfer of relative intensity noise in Brillouin fiber ring lasers," Opt. Lett, vol. 32, no. 1, pp. 11-13, Jan. 2017.

[22] S. Shin, U. Sharma, H. Tu, W. Jung, and S. A. Boppart, "Characterization and analysis of relative intensity noise in broadband optical sources for optical coherence tomography," IEEE Photon. Technol. Lett., vol. 22, no. 14, pp. 1057-1059, Jul. 2010.

[23] A. Minardo, R. Bernini, and L. Zeni, "A simple technique for reducing pump depletion in long-range distributed Brillouin fiber sensors," J. Sensors, vol. 9, no. 6, pp. 633-634, Apr. 2009.

Authors' biography not available at the time of publication. 